This is an author version of the paper:

Basit A., Qureshi W., Dailey M., Krajník T.

Joint Localization of Pursuit Quadcopters and Target Using Monocular Cues

Journal of Intelligent and Robotic Systems, Heidelberg, Springer (2014).

DOI: $10.1007 / \mathrm{s} 10846-014-0081-2$

The final publication is available at Springer websites via

http://dx.doi.org/10.1007/s10846-014-0081-2

\title{
Copyright notice
}

The copyright to the Contribution identified above is transferred to SpringerVerlag GmbH Berlin Heidelberg (hereinafter called Springer-Verlag). The copyright transfer covers the sole right to print, publish, distribute and sell throughout the world the said Contribution and parts thereof, including all revisions or versions and future editions thereof and in any medium, such as in its electronic form (offline, online), as well as to translate, print, publish, distribute and sell the Contribution in any foreign languages and throughout the world. 


\title{
Joint Localization of Pursuit Quadcopters and Target Using Monocular Cues
}

\author{
Abdul Basit • Waqar S. Qureshi . \\ Matthew N. Dailey • Tomáš Krajník
}

Received: date / Accepted: date

\begin{abstract}
Pursuit robots (autonomous robots tasked with tracking and pursuing a moving target) require accurate tracking of the target's position over time. One possibly effective pursuit platform is a quadcopter equipped with basic sensors and a monocular camera. However, combined noise of the quadcopter's sensors causes large disturbances of target's 3D position estimate. To solve this problem, in this paper, we propose a novel method for joint localization of a quadcopter pursuer with a monocular camera and an arbitrary target. Our method localizes both the pursuer and target with respect to a common reference frame. The joint localization method fuses the quadcopter's kinematics and the target's dynamics in a joint state space model. We show that predicting and correcting pursuer and target trajectories simultaneously produces better results than standard approaches to estimating relative target trajectories in a 3D coordinate system. Our method also comprises a computationally efficient visual tracking method capable of redetecting a temporarily lost target. The efficiency of the proposed method is demonstrated by a series of experiments with a real quadcopter pursuing a human. The results show that the visual tracker can deal effectively with target occlusions and that joint localization outperforms standard localization methods.
\end{abstract}

Abdul Basit

Asian Institute of Technology, Klong Luang, Pathumthani (12121), Thailand

Tel.: +66-80-5575717

Fax: +66-2524-5721

E-mail: Abdul.Basit.Khan@ait.asia

Waqar Shahid Qureshi

Asian Institute of Technology, Klong Luang, Pathumthani (12121), Thailand

E-mail: waqar.s.q@ieee.org

Matthew N. Dailey

Asian Institute of Technology, Klong Luang, Pathumthani (12121), Thailand

E-mail: mdailey@ait.asia

Tomáš Krajník

University of Lincoln, Brayford Pool, Lincoln, UK

Dept. of Cybernetics, CTU in Prague, FEE

E-mail: tkrajnik@lincoln.ac.uk 
Keywords Quadcopters · joint localization · monocular cues · state estimation filters · visual tracking $\cdot$ redetection $\cdot$ backprojection · pursuit robot · AR.Drone

\section{Introduction}

Surveillance and monitoring using human operators can be tedious, difficult, dangerous, and error prone. Recent technological developments have enabled the use of mobile robots and vision systems for such applications. Our focus is on mobile robots that are capable of tracking and monitoring a target in scenarios such as person/child/animal monitoring or tracking a fugitive. Ground robots might be useful in some scenarios, but they are expensive and difficult to navigate, as they must avoid obstacles in the field, negotiate uneven surfaces, and place sensors over a sufficient range of heights to get a good view of both the object of interest and the terrain. On the other hand, aerial robots with airborne sensors, which are capable of low-altitude flying and vertical takeoff and land (VTOL) maneuvers, would require less complex navigation and would provide a better field of view. Aerial robots are already being used for other applications such as sports assistance [17], traffic monitoring [14], fire detection [25], remote sensing [18], and precision agriculture $[8,16,15,23,2]$.

Quadcopters are VTOL aerial vehicles with four rotary wings that can hover over a fixed location or perform quick maneuvers but may have limited on-board sensors and processing power due to low payload capacity.

We are investigating the feasibility of autonomous pursuit of targets using inexpensive quadcopters with monocular cameras. We use the Parrot AR.Drone quadcopter (shown in Fig. 1) for research purposes. The AR.Drone is an inexpensive quadcopter with a built-in control system enabling it to hover in a stationary position. For visual tracking, the quadcopter features front-facing and downwardfacing cameras. Localization is aided by an accelerometer, a pressure sensor, a three-axis gyro, and a magnometer. The robot can be teleoperated from a host machine through WiFi.

In pursuit applications where the target's position may be extremely dynamic, the UAV should be capable of fast autonomous planning and motion. It must also be able to track the target object in real time.

In this paper, we take steps toward robust UAV target pursuit. We focus on two important challenges for the monocular vision-based tracker. First, we require an appearance-based tracker that is sufficiently accurate and fast. Second, since such a tracker will necessarily be extremely noisy, we require sensor modeling and state estimation able to obtain target position estimates usable for motion planning.

In the following sections, we introduce related work in $2 \mathrm{D}$ visual target tracking and state estimation for quadcopters and similar UAVs and then we outline our contributions.

\subsection{Localization}

A pursuit robot must keep track of its position and orientation relative to the target and surrounding environment. Localization is a form of sequential state estimation. Sequential sate estimation methods are used extensively in robotics 
and computer vision to solve problems such as SLAM, monocular SLAM [11], visual target tracking [24], and 3D reconstruction. Methods include Kalman filters, particle filters [28], and grid-based methods. The most widely used filtering method that supports non-linear state estimation models is the extended Kalman filter [32].

EKF models are fast and effective in any application in which the posterior state estimate is accurately represented by a Gaussian. The EKF is the first choice as a state filter when error is approximately Gaussian and the non-linearities in the system and sensor are moderate. EKFs have been used by several researchers to improve the performance of visual tracking methods $[29,13,19]$.

Finding the position of a target relative to a UAV using a monocular camera requires extraction of depth cues from the 2D image. Monocular depth cues are extremely noisy, so relying purely on the $2 \mathrm{D}$ image to obtain $3 \mathrm{D}$ positions with depths would introduce a great deal of error. One way to improve the noisy sensor readings we would get from a $2 \mathrm{D}$ visual tracker is to filter based sensor estimates with a sequential state estimator.

In a previous paper [4] we propose a state estimation model based on the EKF for pursuit by SUGVs (Small Unmanned Ground Vehicles) to improve the accuracy of the estimated trajectory of both the pursuit robot and the target. The proposed method fuses robot kinematics and target dynamics to obtain superior robot and target trajectory estimates.

In this paper, based on our previous work, we propose a joint localization model that fuses quadcopter kinematics with target dynamics to improve the relative trajectory estimates of both the quadcopter and the target.

\section{$1.22 \mathrm{D}$ object tracking}

The major methods for 2D image-based tracking use either feature matching, optical flow, or feature histograms. Feature matching algorithms such as SIFT [34], SURF [31], and shape matching algorithms such as contour matching [33] are computationally too expensive to be considered for real-time tracking by a small UAV with modest compute resources. Optical flow methods [12] may be within reach in terms of speed, but they do not maintain an appearance model. Histogram-based trackers, on the other hand, are not only fast, but also maintain an appearance model that is potentially useful for recovering tracking after an occlusion or reappearance in the field of view.

Sliding window is a general approach in computer vision to look for a target object in an image. The naive sliding window approach is computationally inefficient to scan an image to search a target. Porikli [27] propose an "integral histogram" method using integral images to speed up the process. Perreault and Hebert [26] compute histograms for median filtering efficiently by maintaining separate column-wise histograms, and, as the sliding window moves right, first updating the relevant column histogram then adding and subtracting the relevant column histograms to the histogram for the sliding window. Sizintsev et al. [30] take a similar approach to obtain histograms over sliding windows by efficiently updating the histogram using previously calculated histograms for overlapping windows.

CAMSHIFT (Continuously Adaptive Mean Shift) [6,1] is a fast and robust feature histogram tracking algorithm potentially useful for UAVs. The method 


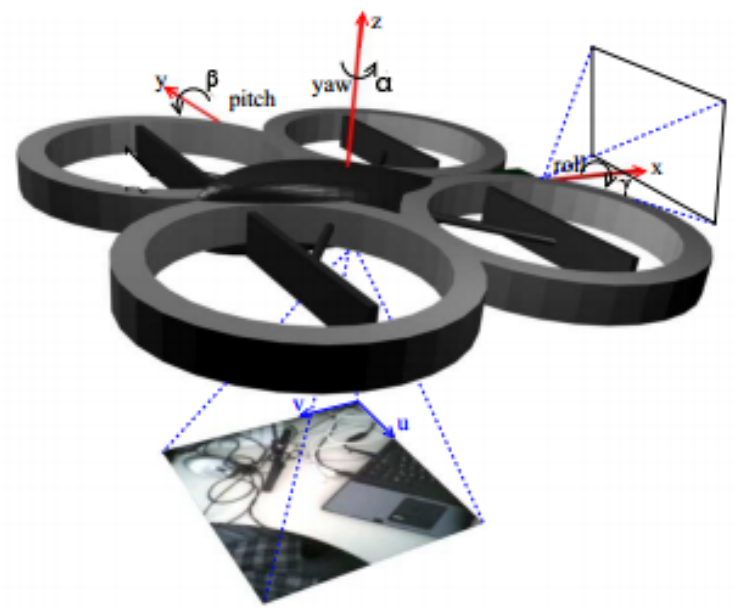

Fig. 1 Quadcopter visual sensors and coordinate system. The AR.Drone quadcopter incorporates a front-facing and downward-facing camera. Roll $\left(\gamma_{t}^{r}\right)$ and pitch $\left(\beta_{t}^{r}\right)$ are used to control forward-backward and left-right linear motions. Overall rotor speed is used to control upwarddownward motion. Yaw control is used for change in orientation. The front-facing camera is useful in applications such as tracking and surveillance. The downward camera is useful for inspecting building, crops, or a field.

begins with manual initialization from a target image patch. It then tracks the region using a combination of color histograms, the basic mean-shift algorithm $[9,10]$, and an adaptive region-sizing step. It is scale and orientation invariant. Unfortunately, since the method performs a search for a local peak in the global backprojection, it is easily distracted by background objects with similar color distributions. In this paper, we incorporate an adaptive histogram similarity threshold with CAMSHIFT to help avoid tracking false targets. We also use this adaptive similarity threshold with a backprojection technique to recover the target object and reinitialize the CAMSHIFT visual tracker after an occlusion.

\subsection{Monocular visual tracking by UAVs}

Bi and Duan [5] implement a visual tracking algorithm using a low-cost quadcopter. The target is a colored landing platform that is moved manually on a cart. The quadcopter is controlled by a host machine that processes the video stream and tracks the landing platform. The host machine calculates the current position of the landing platform by using the centroid of image moments calculated for a binary image that is obtained after thresholding the green channel of the RGB image. The authors use independent controllers for pitch and roll that receive feedback from the visual tracker. The feedback input to the controllers at each frame is the current positioning error.

Kim and Shim [20] present a visual tracker and demonstrate its capabilities and usage on a tablet computer with the AR.Drone. The method uses color and image moments for the visual tracker. 
All of the object tracking and navigation algorithms proposed thus for low cost UAVs use color and image moments. None of this work has demonstrated modelbased object tracking that is sufficiently fast and accurate for real time control of a UAV.

\subsection{Contributions}

In this paper, we propose a novel joint purser and target localization model specifically for the AR.Drone that reduces position estimation error caused by monocular sensor measurements. The model maintains an estimate of the state of the target, assuming a simple linear dynamical model, as well as an estimate of the AR.Drone robot's state, assuming standard quadcopter robot kinematics [7].

We additionally propose a target tracking method suitable for quadcopters that efficiently handles both visual detection and tracking in real time. We perform target redetection when the target is occluded or leaves the field of view. We suspend tracking based on an adaptive histogram threshold. Once the object is reacquired in a redetection phase, we apply CAMSHIFT to confirm the redetected object. After CAMSHIFT validation, we reinitialize the tracker.

We thus fuse information from 2D visual tracking and the UAV's odometry measurements with knowledge of the AR.Drone's kinematics in an extended Kalman filter to obtain superior state estimation. The filter significantly improves estimation accuracy compared to standard sensor-based position estimates as well as compared to localization models not incorporating pursuit robot kinematics.

In an empirical evaluation, we show, on real-world videos, that the proposed method is a robust approach to target tracking and redetection during pursuit that is accurate, is successful at reinitialization, has very low false positive rates, and runs in real time.

\section{System Design}

In this section, we introduce the AR.Drone's hardware (sensors and actuators), provide details of the pursuer-pursuit-target localization method for the AR.Drone, and finally describe visual target tracking method.

\subsection{Quadcopter overview}

The AR.Drone has a carbon fiber support structure and a plastic body with removable hulls optimized for indoor and outdoor flight. The rotors are propelled by high-efficiency brushless DC motors and control circuitry. The drone is equipped with one frontal and one bottom facing camera. The control board of the AR.Drone consists of a 1-GHz ARM-Cortex-A8 32-bit processor with an $800 \mathrm{MHz}$ digital signal processor and 2GB RAM. The user can control the quadcopter's roll, pitch, yaw, and vertical speed through a host machine over a WiFi link. The function of the control board is to convert user navigation commands into motor commands and to adjust the motor speeds to stabilize the drone at the required pose. Fig. 2 shows how the drone communicates with the host machine. We use the video 


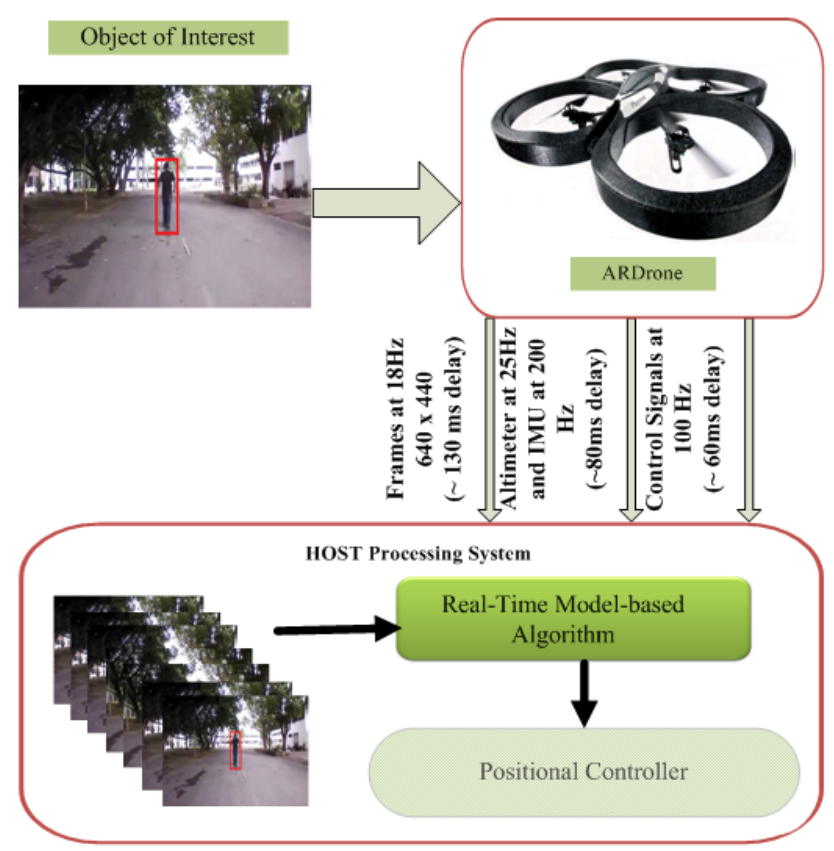

Fig. 2 System architecture and data flow. The base station receives sensor data (images and odometry) at the given rates and latencies. The frequency of the odometry data $(200 \mathrm{~Hz})$ is high compared to the image transfer rate (18 fps), so there is negligible synchronous error in transporting ghostly.

stream from the frontal camera as the main sensor for localization. The camera has a frame rate of approximately $18 \mathrm{fps}$. Estimated roll, pitch, yaw, vertical speed, and velocity in the $x-y$ plane are estimated from the onboard accelerometer, a pressure sensor, a three-axis gyro, and a magnetometer and sent to the host at $200 \mathrm{~Hz}$.

\subsection{Mathematical model and algorithmic flow}

In this section, we detail the joint localization method for the quadcopter purser and target. Joint localization incorporates both target dynamics and drone kinematics to correct the sensor-based measurements of the target's and purser's state. As the main target state measurement sensor, we use the AR.Drone's front-facing monocular camera.

The tracking algorithm's flow is shown in Fig. 3. The monocular 2D visual tracker tracks the target as long as it is in the field of view. The monocular visual tracker returns a 2D tracking window indicating the target's size and position in the image. To transform $2 \mathrm{D}$ measurements into $3 \mathrm{D}$, we take a ray from the camera center through the image plane at the center of the 2D target region and calculate the depth of the target along that ray using the assumed target height in an absolute frame. Meanwhile, we acquire AR.Drone odometry data in the form, at time $t$, of $\left(\gamma_{t}^{r}, \beta_{t}^{r}, \alpha_{t}^{r}, h_{t}^{r}, \dot{x}_{t}^{r}, \dot{y}_{t}^{r}, \dot{z}_{t}^{r}\right)$, where $\left(\gamma_{t}^{r}, \beta_{t}^{r}, \alpha_{t}^{r}\right)$ describes the roll, pitch and yaw of the quadcopter, $h_{t}^{r}$ is the measured altitude, and $\left(\dot{x}_{t}^{r}, \dot{y}_{t}^{r}, \dot{z}_{t}^{r}\right)$ is the 


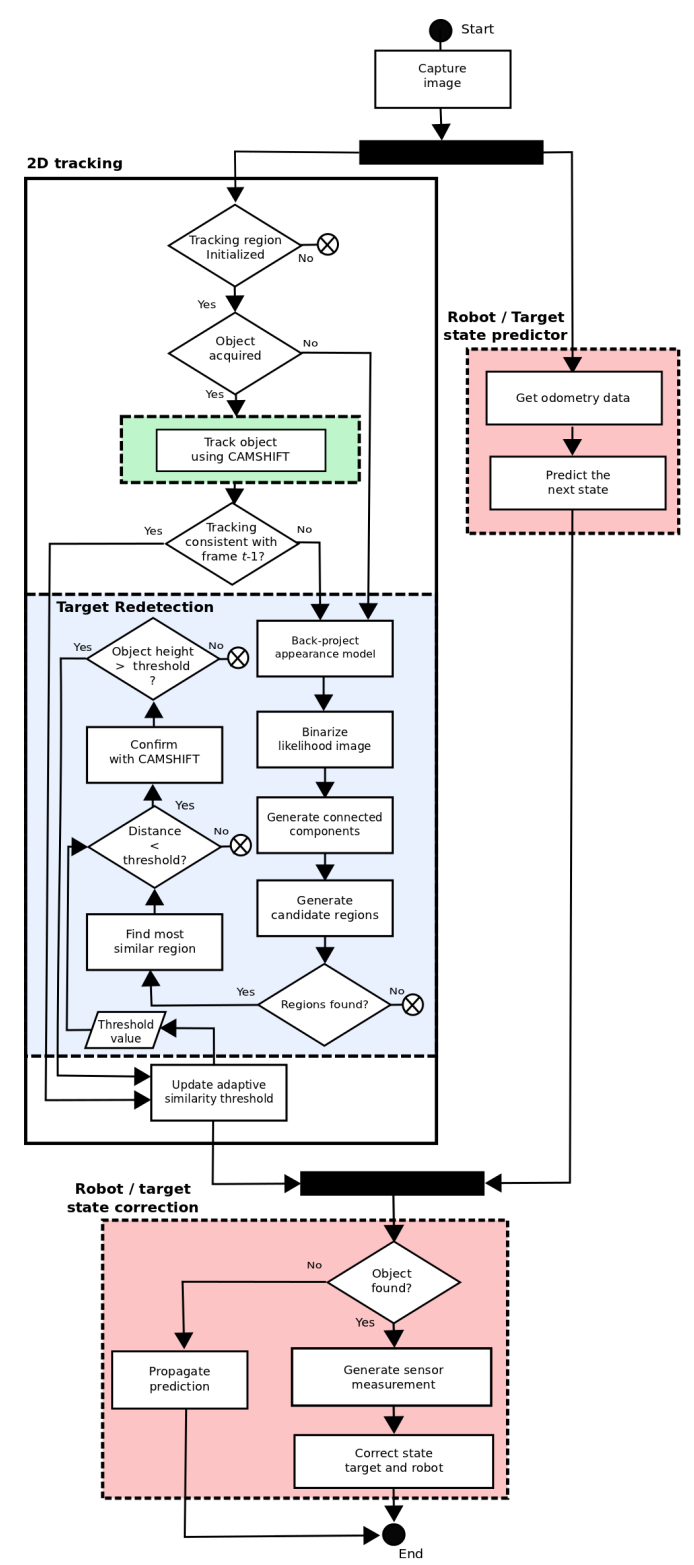

Fig. 3 Algorithm flow. The green box contains the visual target tracking phase of the algorithm. The blue box contains the processing occurring in the redetection phase, when the target is not in the scene. The red box contains localization for fusing odometery and target tracking data.

linear velocity of the quadcopter in the robot coordinate system. See Fig. 4 for visualization of the coordinate system. On the arrival of each image frame, the target tracking sensor measurement and odometry data are fed to the proposed joint localization model. The model predicts the pursuer's and target's new state 
based on the previous state and corrects (updates) the estimate based on the newly acquired sensor measurement. If the target is occluded or leaves the camera field of view, we stop 2D tracking and state correction, and we run a redection algorithm until the target reappears, at which time we restart the normal flow of the algorithm.

We briefly outline the visual tracking method below and provide more details on the 3D state estimate model for the AR.Drone in next section. Refer to Basit et al. [4] for more details on the 2D tracking algorithm, which proceeds as follows:

1. Initialize CAMSHIFT with initial image $I_{0}$ and bounding box $\left(x_{c}, y_{c}, w, h\right)$.

2. Continue CAMSHIFT tracking, updating adaptive histogram threshold.

3. Suspend tracking when similarity between region returned by the tracker and appearance model is too low.

4. Run redetection.

5. Suspend redectection when candidate target most similar to the appearance model is similar and large enough.

6. Run CAMSHIFT to confirm redetected region. If confirmed, reinitialize tracking; otherwise, return to redetection.

\subsection{Joint Estimation of AR.Drone and Target State}

In this section we describe the proposed model for obtaining smooth pursuer and target trajectories in the 3D world coordinate frame.

\subsubsection{System state}

The system state expresses the UAV's position and the target's position and velocity in the world coordinate frame. We define the system state at time $t$ to be

$$
\mathbf{x}_{t}=\left[x_{t}, y_{t}, z_{t}, \dot{x}_{t}, \dot{y}_{t}, \dot{z}_{t}, x_{t}^{r}, y_{t}^{r}, z_{t}^{r}, \gamma_{t}^{r}, \beta_{t}^{r}, \alpha_{t}^{r}\right]^{T},
$$

where $\left(x_{t}, y_{t}, z_{t}\right)$ is the target's position, $\left(\dot{x}_{t}, \dot{y}_{t}, \dot{z}_{t}\right)$ is the target's velocity, $\left(x_{t}^{r}, y_{t}^{r}, z_{t}^{r}\right)$ is the pursuer's position, and $\left(\gamma_{t}^{r}, \beta_{t}^{r}, \alpha_{t}^{r}\right)$ is the pursuer's 3D orientation (roll, pitch and, yaw) respectively. The positions and orientations are expressed in the world coordinate frame. The state transition model is defined as

$$
\mathbf{x}_{t+1}=\mathbf{f}\left(\mathbf{x}_{t}, \mathbf{u}_{t}\right)+\nu_{t},
$$

where $\nu_{t} \sim \mathcal{N}\left(\mathbf{0}, \mathbf{Q}_{t}\right)$. $\mathbf{f}\left(\mathbf{x}_{t}, \mathbf{u}_{t}\right)$ has two components. The first component models the AR.Drone's kinematics, assuming constant linear and angular velocity over short time periods (acceleration is modeled as noise). The second component is a first order linear dynamical system for the target's motion. We describe each component in turn. The odometry control vector is

$$
\mathbf{u}_{t}=\left[\begin{array}{llll}
\delta \gamma_{t}^{r} & \delta \beta_{t}^{r} & \delta \alpha_{t}^{r} & \delta h_{t}^{r}
\end{array}\right]^{T},
$$

defining the change in the roll $\left(\gamma_{t}^{r}\right)$, pitch $\left(\beta_{t}^{r}\right)$, yaw $\left(\alpha_{t}^{r}\right)$, and altitude $\left(h_{t}^{r}\right)$ of the quadcopter at time $t$. 


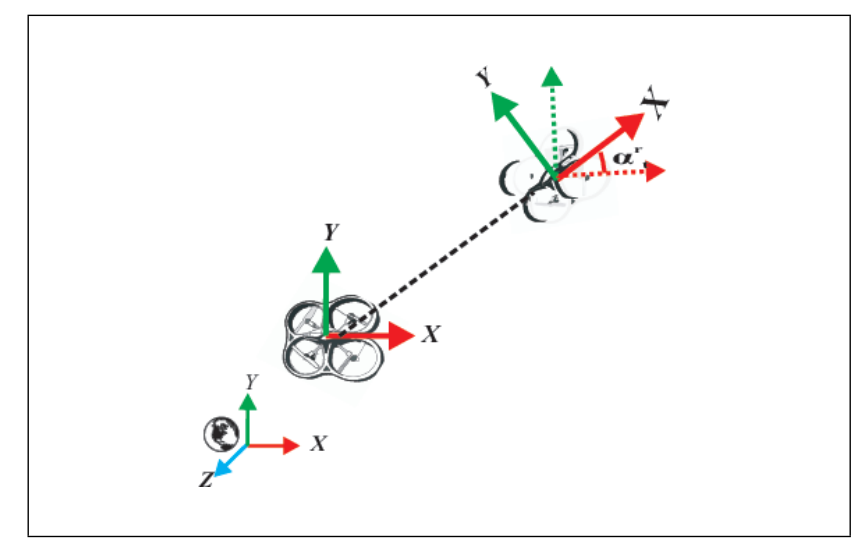

Fig. 4 Birds-eye view of quadcopter motion in 3D world coordinate frame. The motion depends on changes of roll, pitch and altitude controls, that in turn produce 3D linear and angular motion. Linear velocities measurements $\left(\dot{x}_{t}^{r}, \dot{y}_{t}^{r}, \dot{z}_{t}^{r}\right)$ are provided by three sensors. The yaw $\left(\alpha_{t}^{r}\right)$ angle is used to change the orientation of the quadcopter and also help to convert velocities into world coordinate frame.

The state transition for the AR.Drone is then assumed to be

$$
\begin{aligned}
x_{t+1}^{r} & =x_{t}^{r}+\left(\dot{x}_{t}^{r} \cos \alpha_{t}^{r}-\dot{y}_{t}^{r} \sin \alpha_{t}^{r}\right) \cdot \Delta_{t} \\
y_{t+1}^{r} & =y_{t}^{r}+\left(\dot{x}_{t}^{r} \sin \alpha_{t}^{r}+\dot{y}_{t}^{r} \cos \alpha_{t}^{r}\right) \cdot \Delta_{t} \\
z_{t+1}^{r} & =z_{t}^{r}+\delta h_{t}^{r} \\
\gamma_{t+1}^{r} & =\gamma_{t}^{r}+\delta \gamma_{t}^{r} \\
\beta_{t+1}^{r} & =\beta_{t}^{r}+\delta \beta_{t}^{r} \\
\alpha_{t+1}^{r} & =\alpha_{t}^{r}+\delta \alpha_{t}^{r},
\end{aligned}
$$

where $\left(\dot{x}_{t}^{r}, \dot{y}_{t}^{r}\right)$ is the linear velocity of the quadcopter in the $(x, y)$ plane. This velocity depends on the quadcopter's roll and pitch in the world coordinate frame. See Fig. 5. We define the dynamics

$$
\begin{aligned}
& \dot{x}_{t+1}^{r}=\mathrm{C}_{1} \beta_{t}^{r} \\
& \dot{y}_{t+1}^{r}=\mathrm{C}_{2} \gamma_{t}^{r}
\end{aligned}
$$

To transform the linear velocities from the UAV frame to the world coordinate frame, we have

$$
\mathbf{v}_{t}^{w}=\mathrm{R}_{t} \dot{\mathbf{v}}_{t}^{r}
$$

where $\dot{\mathbf{v}}_{t}^{r}$ is simply

$$
\dot{\mathbf{v}}_{t}^{r}=\left[\begin{array}{lll}
\dot{x}_{t}^{r} & \dot{y}_{t}^{r} & \dot{z}_{t}^{r}
\end{array}\right]^{T}
$$

and $\mathrm{R}_{t}$ is 


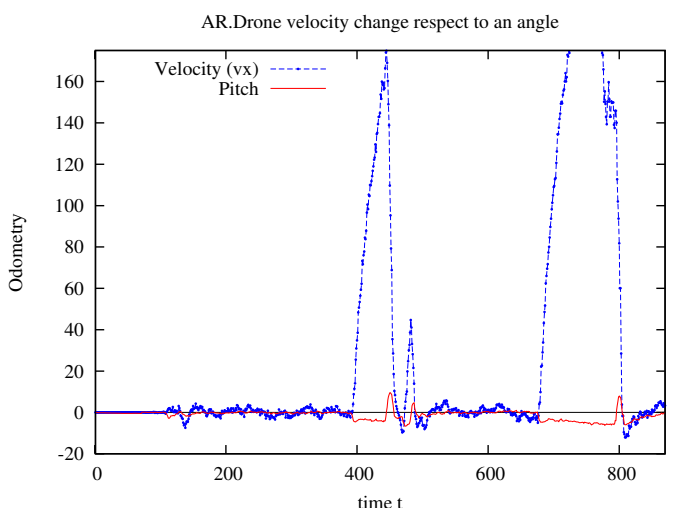

(a)

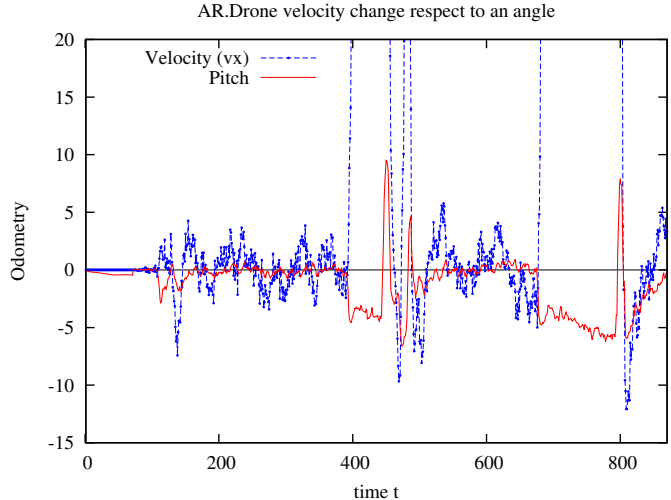

(b)

Fig. 5 (a). Change in linear velocity $\dot{x}_{t}$ with respect to change in pitch angle $\beta_{t}$. Velocity $\dot{x}_{t}$ is in $\mathrm{cm}$ whereas pitch is shown in radians. (b). Zoom image of image (a), by setting y-range $[-15 \cdots 20]$.

$$
\mathrm{R}_{t}=\left[\begin{array}{ccc}
\mathrm{c}_{\alpha_{t}^{r}} \mathrm{c}_{\beta_{t}^{r}} & \mathrm{c}_{\alpha_{t}^{r}} \mathrm{~S}_{\beta_{t}^{r}} \mathrm{~S}_{\gamma_{t}^{r}}-\mathrm{S}_{\alpha_{t}^{r}} \mathrm{c}_{\gamma_{t}^{r}} & \mathrm{c}_{\alpha_{t}^{r}} \mathrm{~S}_{\beta_{t}^{r}} \mathrm{c}_{\gamma_{t}^{r}}+\mathrm{s}_{\alpha_{t}^{r}} \mathrm{~S}_{t}^{r} \\
\mathrm{~S}_{\alpha_{t}^{r}} \mathrm{c}_{\beta_{t}^{r}} & \mathrm{~S}_{\alpha_{t}^{r}} \mathrm{~S}_{\beta_{t}^{r}} \mathrm{~S}_{\gamma_{t}^{r}}+\mathrm{c}_{\alpha_{t}^{r}} \mathrm{c}_{\gamma_{t}^{r}} \mathrm{~S}_{\alpha_{t}^{r}} \mathrm{~S}_{\beta_{t}^{r}} \mathrm{c}_{\gamma_{t}^{r}}-\mathrm{c}_{\alpha_{t}^{r}} \mathrm{~S}_{t}^{r} \\
-\mathrm{S}_{\beta_{t}^{r}} & \mathrm{c}_{\beta_{t}^{r}} \mathrm{~S}_{t}^{r} & \mathrm{c}_{\beta_{t}^{r}} \mathrm{c}_{\gamma_{t}^{r}}
\end{array}\right],
$$

where c. and s. are shorthand for the cosine and sine functions.

Target motion We assume a simple linear dynamics

$$
\begin{aligned}
x_{t+1} & =x_{t}+\Delta_{t} \dot{x}_{t} \\
y_{t+1} & =y_{t}+\Delta_{t} \dot{y}_{t} \\
z_{t+1} & =z_{t}+\Delta_{t} \dot{z}_{t} \\
\dot{x}_{t+1} & =\dot{x}_{t} \\
\dot{y}_{t+1} & =\dot{y}_{t} \\
\dot{z}_{t+1} & =\dot{z}_{t}
\end{aligned}
$$

for the target object's state. 
Linearization Since $\mathbf{f}\left(\mathbf{x}_{t}, \mathbf{u}_{t}\right)$ is nonlinear and we will be using an extended Kalman filter, we must approximate the system described in Eq. 2 by linearizing around an arbitrary point $\hat{\mathbf{x}}_{t}$. We write

$$
\mathbf{f}\left(\mathbf{x}_{t}, \mathbf{u}_{t}\right) \approx \mathbf{f}\left(\hat{\mathbf{x}}_{t}, \mathbf{u}_{t}\right)+\mathrm{J}_{f_{t}}\left(\mathbf{x}_{t}-\hat{\mathbf{x}}_{t}\right)
$$

where $\mathrm{J}_{f_{t}}$ is the Jacobian

$$
\mathrm{J}_{f_{t}}=\left[\frac{\partial \mathbf{f}\left(\mathbf{x}_{t}, \mathbf{u}_{t}\right)}{\partial \mathbf{x}_{t}}\right]
$$

evaluated at $\hat{\mathbf{x}}_{t}$. We omit the detailed Jacobian calculations.

\subsubsection{Sensor model}

We assume the quadcopter's target tracking camera is mounted, in a fixed position near the UAV's center of rotation with roll (rotation around the principal axis) close to 0 . We also incorporate a $2 \mathrm{D}$ visual tracking algorithm, capable of producing an estimate of the $2 \mathrm{D}$ position of the object's projection into the image plane at time $t$. We assume the operator initially defines the target object to pursue by specifying a bounding box around it in the first frame. We use visual tracker based on CAMSHIFT to track the object from frame to frame.

The measurement from the algorithm is thus simply a bounding box:

$$
\mathbf{z}_{t}=\left[u_{t}, v_{t}, w_{t}^{i m g}, h_{t}^{i m g}\right]^{T}
$$

where $\left(u_{t}, v_{t}\right)$ is the center and $w_{t}^{i m g}$ and $h_{t}^{i m g}$ are the width and height of the bounding box in the image. We model the sensor with a function $\mathbf{h}(\cdot)$ mapping the system state $\mathbf{x}_{t}$ to the corresponding sensor measurement

$$
\mathbf{z}_{t}=\mathbf{h}\left(\mathbf{x}_{t}\right)+\zeta_{t}
$$

with $\zeta \sim \mathcal{N}\left(\mathbf{0}, \mathrm{S}_{t}\right)$.

For a pinhole camera with focal length $f$ and principal point $\left(c_{x}, c_{y}\right)$, ignoring the negligible in-plane rotation of the cylindrical object, we can write target's center $\left(u_{t}, v_{t}\right)$ and size $\left(w_{t}^{i m g}, h_{t}^{i m g}\right)$

$$
\begin{aligned}
u_{t} & =\left(f x_{t}^{c a m}+c_{x}\right) / z_{t}^{c a m} \\
v_{t} & =\left(f y_{t}^{c a m}+c_{y}\right) / z_{t}^{c a m} \\
w_{t}^{i m g} & =f w_{0} / z_{t}^{c a m} \\
h_{t}^{i m g} & =f h_{0} / z_{t}^{c a m},
\end{aligned}
$$

where $\left(h_{0}, w_{0}\right)$ is the assumed target's height and width, $\left(x_{t}^{c a m}, y_{t}^{c a m}, z_{t}^{c a m}\right)$ is the is the center of the target in the camera coordinate system and its homogeneous representation is

$$
\mathbf{x}_{t}^{c a m}=\mathrm{T}_{t}^{W / C}\left[\begin{array}{c}
x_{t} \\
y_{t} \\
z_{t} \\
1
\end{array}\right]
$$


where the transformation $\mathrm{T}_{t}^{W / C}$ is defined as

$$
\mathrm{T}_{t}^{W / C}=\mathrm{T}^{R / C} \mathrm{~T}_{t}^{W / R}
$$

where $\mathrm{T}_{t}^{W / R}$ is the rigid transformation from the world coordinate system to the robot coordinate at time $t$, and $\mathrm{T}^{R / C}$ is the (fixed) transformation from the robot coordinate system to the camera coordinate system. Expressing the AR.Drone orientation by a rotation matrix $\mathrm{R}_{t}^{T}$ at time $t$, we can write the transformation matrix $\mathrm{T}^{W / R}$

$$
\mathrm{T}_{t}^{W / R}=\left[\begin{array}{cc}
\mathrm{R}_{t}^{T} & -\mathrm{R}_{t}^{T} \mathbf{x}_{t}^{r} \\
\mathbf{0}^{T} & 1
\end{array}\right],
$$

where $\mathbf{x}_{t}^{r}=\left[\begin{array}{lll}x_{t}^{r} & y_{t}^{r} & z_{t}^{r}\end{array}\right]$.

As with the transition model, to linearize $\mathbf{h}\left(\mathbf{x}_{t}\right)$ around an arbitrary point $\hat{\mathbf{x}}_{t}$, we require the Jacobian

$$
\mathrm{J}_{h_{t}}=\left[\frac{\partial \mathbf{h}\left(\mathbf{x}_{t}\right)}{\partial \mathbf{x}_{t}}\right]
$$

evaluated at an arbitrary point $\hat{\mathbf{x}}_{t}$.

\subsubsection{Initialization}

We require an a-priori state vector $\mathbf{x}_{0}$ to initialize the system. As explained before, we assume that the quadcopter is at the origin of the world coordinate system or an alternative initial position of the robot is given. We do not assume any knowledge of the target's initial trajectory. We can therefore treat the user-provided initial target bounding box as a first sensor measurement $\mathbf{z}_{0}$ and initialing the system as

$$
\hat{\mathbf{x}}_{0}=\left[x_{0}, y_{0}, z_{0}, 0,0,0,0,0,0,0,0,0\right]^{T}=\mathbf{h}^{i n v}\left(\mathbf{z}_{0}\right)
$$

To obtain $\left(x_{0}, y_{0}, z_{0}\right)$, we first calculate an initial target position in the cameracoordinate frame $\mathbf{x}_{0}^{\text {cam }}$ then, noting that the robot frame at time 0 is also the world frame, we can map to the world coordinate frame by

$$
\left[\begin{array}{c}
x_{0} \\
y_{0} \\
z_{0} \\
1
\end{array}\right]=\left(\mathrm{T}^{R / C}\right)^{-1}\left[\begin{array}{c}
x_{0}^{c a m} \\
y_{0}^{c a m} \\
z_{0}^{c a m} \\
1
\end{array}\right]
$$

Inspecting the system in Eq. 13, we can find $x_{0}^{c a m}$ and $y_{0}^{\text {cam }}$ given $u_{t}$ and $v_{t}$ if $z_{0}^{\text {cam }}$ is known. We can obtain $z_{0}^{\text {cam }}$ from $w_{0}^{i m g}$ or $h_{0}^{i m g}$. We use $z_{0}^{i m g}=f \cdot h / h_{0}^{i m g}$ on the assumption that the user-specified bounding box is more accurate vertically than horizontally. 


\subsubsection{Noise parameters}

The sensor noise is given by the matrix $\mathrm{S}_{t}$. We assume that the measurement noise for both the bounding box center and the bounding box size are a fraction of the target's width and height in the image:

$$
\mathrm{S}_{t}=\lambda^{2}\left[\begin{array}{cccc}
\left(w_{t}^{i m g}\right)^{2} & 0 & 0 & 0 \\
0 & \left(h_{t}^{i m g}\right)^{2} & 0 & 0 \\
0 & 0 & \left(w_{t}^{i m g}\right)^{2} & 0 \\
0 & 0 & 0 & \left(h_{t}^{i m g}\right)^{2}
\end{array}\right]
$$

We use $\lambda=0.1$ in our simulation that is $10 \%$ of the target width and height represented by $2 \mathrm{D}$ bounding box in image. We receive this number after conducting series of experiments with CAMSHIFT visual tracker in real environment. For the initial state error denoted by $\mathrm{P}_{0}$, we propagate the measurement error for $\mathbf{z}_{0}$ through $\mathbf{h}^{i n v}\left(\mathbf{z}_{0}\right)$ and take into account the initial uncertainty about the target's velocity:

$$
\mathrm{P}_{0}=\mathrm{J}_{h^{i n v}} \mathrm{~S}_{0} \mathrm{~J}_{h^{i n v}}+\operatorname{diag}(0,0,0, \eta, \eta, \eta, 0,0,0,0,0,0),
$$

$\eta$ is a constant and $\mathrm{J}_{h^{i n v}}$ is the Jacobian of $\mathbf{h}^{i n v}(\cdot)$ evaluated at $\mathbf{z}_{0}$.

We assume that the state transition noise covariance $Q_{t}$ is diagonal for simplicity. We tie the covariance $Q_{t}$ to the target linear velocities $\left(\dot{x}_{t}, \dot{y}_{t}, \dot{z}_{t}\right)$, AR.Drone linear velocities $\left(\dot{x}_{t}^{r}, \dot{y}_{t}^{r}, \dot{z}_{t}^{r}\right)$ and orientation $\left(\gamma_{t}^{r}, \beta_{t}^{r}, \alpha_{t}^{r}\right)$ odometry reading. We let

$$
\left[\begin{array}{l}
v_{t} \\
s_{t}
\end{array}\right]=\left[\begin{array}{c}
\sqrt{\dot{x}_{t}^{2}+\dot{y}_{t}^{2}+\dot{z}_{t}^{2}} \\
\sqrt{\left(\gamma_{t}^{r}\right)^{2}+\left(\beta_{t}^{r}\right)^{2}+\left(\delta \alpha_{t}^{r}\right)^{2}}
\end{array}\right],
$$

We let the entries of $\mathrm{Q}_{t}$ corresponding to the target position be $\Delta_{t}^{2}\left(\rho_{1} v_{t}^{2}+\rho_{2}\right)$ and the entries of $\mathrm{Q}_{t}$ corresponding to the target velocity be $\Delta_{t}^{2}\left(\rho_{3} v_{t}^{2}+\rho_{4}\right)$. We let the entries of $\mathrm{Q}_{t}$ corresponding to the AR.Drone's position be $\Delta_{t}^{2}\left(\rho_{5} s_{t}^{2}+\rho_{6}\right)$, and we let the entries of $\mathrm{Q}_{t}$ corresponding to the robot's orientation be $\Delta_{t}^{2}\left(\rho_{7} s_{t}^{2}+\rho_{8}\right)$. This noise distribution is overly simplistic and may ignore some factors, but it is sufficient for the experiments reported in this paper. In total, there are nine free parameters $\left(\eta, \rho_{1}, \rho_{2}, \cdots, \rho_{8}\right)$ that must be determined through hand tuning or calibration. In our simulation, we find optimal parameters free using gradient decent.

\subsubsection{Update algorithm}

Given all the preliminaries specified in the previous sections, the update algorithm is just the standard extended Kalman filter, with modification to handle cases where the color region tracker fails due to occlusions or the target leaving the field of view. When no sensor measurement $\mathbf{z}_{t}$ is available, we simply predict the system state and allow diffusion of the state covariance without sensor measurement correction. When we do have a sensor measurement but the estimated state is far from the predicted state, we reset the filter, using the existing robot position and orientation but fixing the relative target state to that predicted by $\mathbf{h}^{\text {inv }}\left(\mathbf{z}_{t}\right)$ and fixing the elements of $\mathrm{P}_{t}$ by propagating the sensor measurement error for $\mathbf{z}_{t}$ through $\mathbf{h}^{i n v}\left(\mathbf{z}_{t}\right)$ as previously explained in Section 2.3.4. Here is a summary of the algorithm: 
1. Input $\mathbf{z}_{0}$.

2. Calculate $\hat{\mathbf{x}}_{0}$ and $\mathrm{P}_{0}$.

3. For $t=1, \ldots, T$, do

(a) predict $\hat{\mathbf{x}}_{t}^{-}=\mathbf{f}\left(\hat{\mathbf{x}}_{t-1}, \mathbf{u}_{t-1}\right)$,

(b) calculate $\mathrm{J}_{f_{t}}$ and $\mathrm{Q}_{t}$,

(c) predict $\mathrm{P}_{t}^{-}=\mathrm{J}_{f_{t}} \mathrm{P}_{t-1} \mathrm{~J}_{f_{t}}^{T}+\mathrm{Q}_{t}$.

(d) If $\mathbf{z}_{t}$ is unavailable,

i. let $\hat{\mathbf{x}}_{t}=\hat{\mathbf{x}}_{t}^{-}$,

ii. let $\mathrm{P}_{t}=\mathrm{P}_{t}^{-}$.

(e) Otherwise

i. calculate $\mathrm{J}_{h_{t}}, \mathrm{~S}_{t}$, and Kalman gain $\mathrm{K}_{t}=\mathrm{P}_{t}^{-} \mathrm{J}_{h_{t}}^{T}\left(\mathrm{~J}_{h_{t}} \mathrm{P}_{t}^{-} \mathrm{J}_{h_{t}}^{T}+\mathrm{S}_{t}\right)^{-1}$,

ii. estimate $\hat{\mathbf{x}}_{t}=\hat{\mathbf{x}}_{t}^{-}+\mathrm{K}_{t}\left(\mathbf{z}_{t}-\mathbf{h}_{t}\left(\hat{\mathbf{x}}_{t}^{-}\right)\right)$,

iii. update the error estimate $\mathrm{P}_{t}=\left(\mathrm{I}-\mathrm{K}_{t} \mathrm{~J}_{h_{t}}\right) \mathrm{P}_{t}^{-}$.

(f) If $\left\|\hat{\mathbf{x}}_{t}-\hat{\mathbf{x}}_{t}^{-}\right\|>\sigma$, reset the filter.

\section{Experimental setup}

We tested the performance and efficacy of the proposed method by carrying out experiments, not only on synthetic data, but also in real-world scenarios. Before deploying to the real AR.Drone, we performed a simulation to test model correctness and effectiveness. We then carried out real-world experiments in both indoor and outdoor environments. We compare the proposed joint estimation model with two baselines: 1) relative localization without any filtering, and 2) relative localization with Kalman filter-based sensor correction but no joint estimation of purser and target state. We analyze the data quantitatively for indoor and qualitatively for outdoor environments.

For the simulation experiment, we generated pose data without any noise both for the AR.Drone and target object. The data consist of the target's simulated position with appropriate velocity and simulated AR.Drone's odometry (orientation and linear velocities). We used these as ideal ground truth trajectories. We then produced noisy simulated odometry readings. In order to generate noisy trajectories, we added noise to the AR. Drone odometry data and to the $2 \mathrm{D}$ target bounding box. Direct calculation of pursuer and targer positions from the noisy odometry and noisy target bounding boxes gives us baseline method 1, i.e., sensor-only relative localization without filtering. We simulated a camera generating $640 \times 480$ images at $20 \mathrm{fps}$ with a focal length of 550 pixels (horizontal field of view $60^{\circ}$ ).

For the real-world experiments, we performed quantitative evaluation in indoor environments, where we acquired ground truth data for the target and purser using circular markers pasted on the two objects. The circular markers were of a known diameter. We use a fixed, calibrated camera to track the markers in order to estimate, as accurately as possible, real-world ground truth for both the target and the AR.Drone using Krajník and Nitsche method [22]. See Fig. 6 for a photo of settings. We use these ground truth for computing root mean square error (RMSE). We also tested the proposed visual tracker with the AR.Drone's front facing monocular camera in this indoor environment.

The outdoor environment is a more desirable environment for testing the proposed methods for joint localization and visual tracker with the AR.Drone. How- 


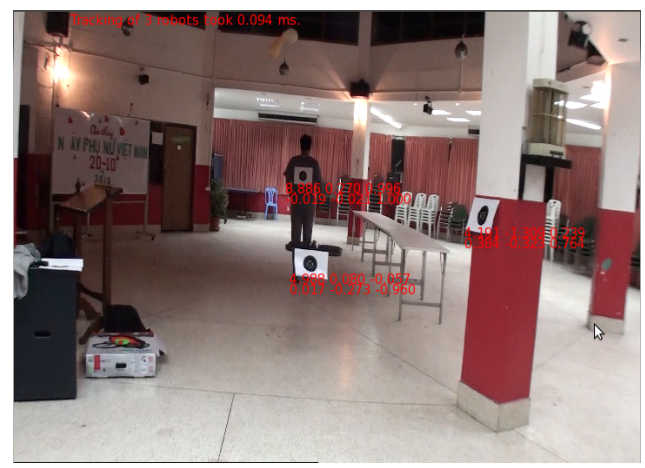

Fig. 6 Markers on the target and AR.Drone localizing their world-coordinate position with reference to an additional calibrated camera.

ever, we are deprived of ground truth data because we have tracked the target for a longer period of time and the fixed camera was unable to keep the markers in its field of view for the entire experiment duration. Therefore, we carried out a qualitative outdoor evaluation in which we tested the stability and smoothness of the proposed method's estimates.

In both indoor and outdoor environments, the target object was a person wearing distinctive clothing and a background containing trees, grass, chairs, shadows, and diffuse light. The target moved with varying velocities and directions and was followed by the AR.Drone under teleoperation. The operator adjusted the drone's position to follow target as smoothly as possible. Since the goal of this paper is to asses localization only, we did not use an autonomous position controller for the pursuer; this module will be assessed in future work.

Concerning the visual tracker, we have already reported speed and accuracy tests for the algorithm on different platforms with different hand-held cameras [3]. In this paper we test the proposed visual tracker specifically on the front-facing monocular camera of the AR.Drone, both in indoor and outdoor environments. Additionally we test our the method with online pursuit video sequence by Klein et al. [21], the result shows how the proposed method recover occlusion.

\section{Results}

In this section, we provide detailed results and analysis for each experiment outlined in the previous section.

We define two baseline methods as points of comparison with the proposed joint state estimation method. The first baseline method does not use any filter while tracking the target $[6,1]$; the sensor-based relative target position estimate is simply accepted. Hence it is named "Localization with no filter correction." The second baseline uses sensor measurement correction based on an ordinary extended Kalman filter [?] to smooth the target's estimated trajectory in the pursuer robot's coordinate system. This baseline is named "Localization with sensor correction." I do not explicitly incorporate the pursuer's odometry measurements either jointly or separately in the baseline methods. The proposed method, however, jointly corrects the pursuer's odometry with the sensor measurement to produce better 


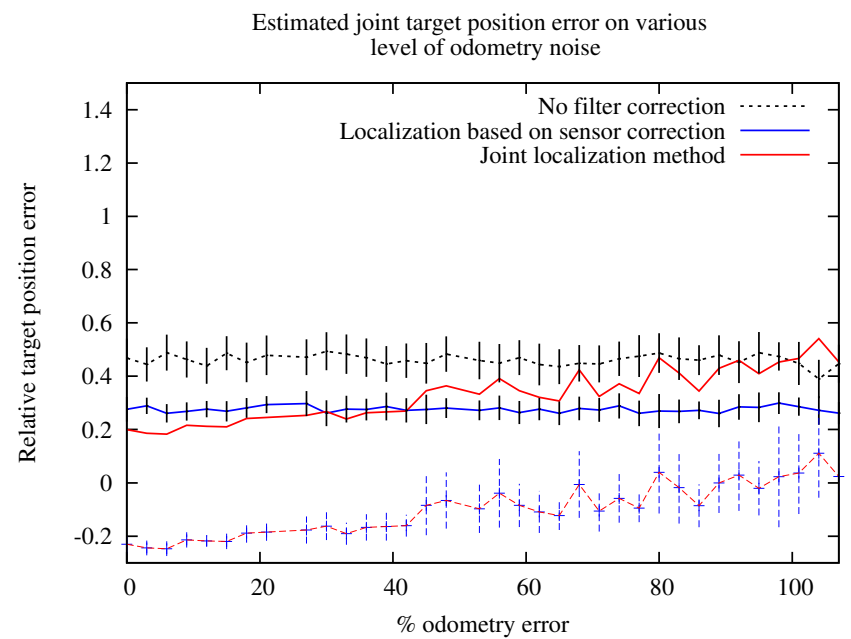

Fig. 7 Experiment I: We simulated the pursuit robot with different levels of odometry noise and measured the average relative target position error for the three estimation methods. Error bars denote $95 \%$ confidence intervals.

performance. The proposed joint localization integrate target and robot pose in a joint state space model, shown in Eq 1."

\subsection{Experiment I: Joint localization simulation}

First we demonstrate the efficacy of fusing target dynamics and AR.Drone kinematics in one state space model in a simulation experiment. We simulated scenarios containing different levels of AR.Drone odometry noise, while keeping the sensor noise constant. The noise ranged from $0 \%$ to $110 \%$. The proposed method shows significant improvement over the baseline methods so long as noise level is below $30 \%$. Performance deteriorates above the $40 \%$ noise level. The performance of the other two methods remains constant over odometry noise levels because they do not use odometry data.

We also evaluated the joint localization model with synthetic data in which a moving UAV chased a moving target. At each iteration of localization the RMSE is computed between the estimated and ground truth target positions in the robot coordinate frame, using each of the three methods. We also suspend joint localization when visual tracker suspend target tracking and wait for the detector to redetect the target and reinitialize the visual tracker that also reinitialize the joint localization. See the first graph of Fig. 8 (c). The proposed method provides more stable and smooth estimates than the baseline methods.

We performed statistical tests on the RMSE of each method compared to ground truth for odometry noise of $15 \%$. The result is shown in the bottom of the Fig. 8 (c). The proposed method outperforms the existing methods by $65 \%$ and $25 \%$. 


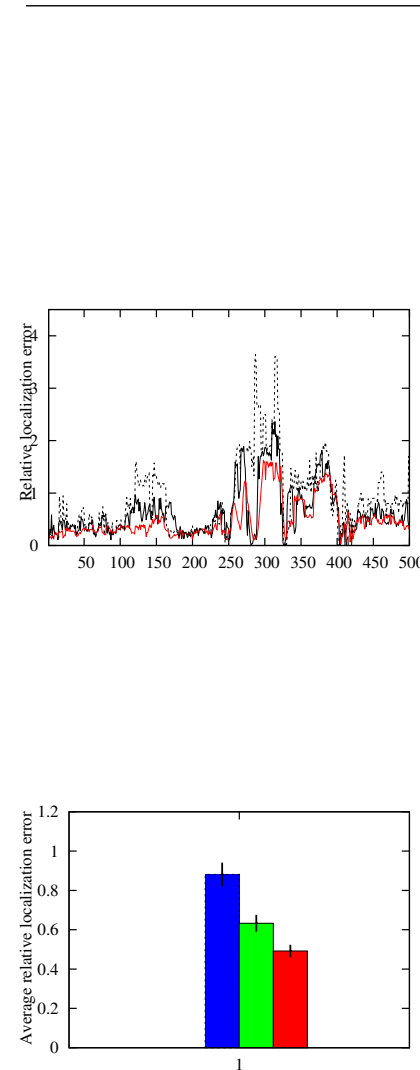

(a) indoor - large open room

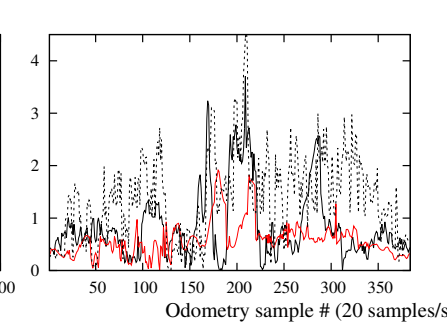

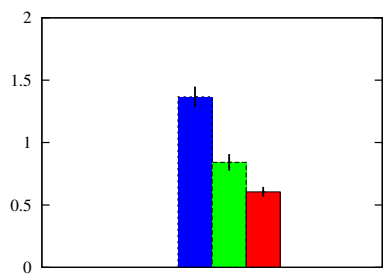

(b) indoor - corridor sensor correction Joint localization

Fig. 8 Experiment I and II: Quantitative evaluation with real world (indoor) and synthetic data. First row shows relative target position root mean squared error (RMSE) of the target in the robot coordinate frame. Second row reflects accumulated average error with $95 \%$ confidence interval. (a) Real world indoor experiment in closed hall. The proposed method is smooth and stable, $44 \%$ and $22 \%$ better than other two methods. (b) Indoor experiment in a corridor. RMSE is lower $56 \%$ and $28 \%$ than other methods (c) Simulation with synthetic data. Propose method outperforms $75 \%$ tracking method without model-based correction and $27 \%$ sensorbased correction.

\subsection{Experiment II: Indoor joint localization}

In experiment II, we carried out indoor experiments with quantitative analysis. We used ground truth data as for comparison between the proposed and existing methods.

At every iteration of each algorithm, at time $t$, we transformed the target position $\left(x_{t}, y_{t}, z_{t}\right)$ to the AR.Drone coordinate reference frame. We calculated root mean squared error between each method's estimate and ground truth. See Fig. 8 (a) and 8 (b) top row. We collapse the results multiple indoor runs into two cases, a large open room and a corridor.

The proposed method clearly obtains smoother and more stable estimates compared to the baseline methods. Although relative localization with sensor correction produces better results when compared with localization with no filter, our 
joint localization method, fusing sensor measurements and odometry data, produces additional improvement.

As with the simulation data, we computed the average root mean square error over all runs. The results is shown in the bottom row of Fig. 8 (a) and 8 (b). The results show that the filtering methods help to smooth the raw sensor measurements, but the proposed joint localization method outperforms not only the method with no filter but also relative localization with sensor correction. Joint localization improves relative estimation by relative to the first baseline and relative to the second baseline method.

\subsection{Experiment III: Outdoor joint localization}

As mentioned earlier, though quantitative analysis is not possible outdoors, we did perform an outdoor experiment to ensure the practicality of the proposed approach. We evaluated our method at several stages of the visual tracking and localization process. First we show results when the target is standing still and the UAV is simply hovering. See Fig. 9 (a). Next we show results when the target is moving with variable velocity and the UAV is accelerating to catch up with the target. See Fig. 9 (b). Finally, we show results when sensor noise increases due to changes in background and lighting. See Fig. 9 (c).

Refer to Fig. 9 (a). The estimated $X$ position varies between 5.5 and 7.5 meters, a range of 2 meters. Recall from $X$ Fig. 1 that the target's position is the distance of the target from the AR.Drone in the forward direction. The proposed method's estimated trajectory, shown in red, is smoother and has lower variance than that of the other methods. The spikes in the trajectory reflect noisy variation in the estimated 2D bounding box's size. The joint localization method infers smooth transitions between neighbouring intervals. The estimated $Y$ position varies between 0 and 0.4 meters. The variation is lower on this axis, reflecting lower noise in the estimated left-right position of the $2 \mathrm{D}$ bounding box. The proposed method again performs better than the baseline methods. Finally the estimated $Z$ position varies between -0.5 and -1.2 meters. We observe more spikes or jerking movements on this axis, generally caused by the UAV's pitch control. The $Z$ position estimates are again smoother than for other baseline methods. Overall, the joint localization method clearly performs better than the baseline methods.

Next, refer to Fig. 9 (b). During this period, the target is moving away from AR.Drone which is attempting to catch up. The AR.Drone begins accelerating at $t=200$ and decelerating at $t=265$. The estimated $X$ position varies between 5.5 and 8.0 meters. The large black-dotted spike indicates extreme sensor noise when the AR.Drone started decelerating. $\dot{x}_{t}^{r}$ is minimized when the pitch $\beta_{t}^{r}=0$. The same spike can be observed in the $Y$ target position in the robot coordinate frame. Here the noise varies between 0 and 0.6 meters. Clearly the proposed method is smoother and more stable along both the $X$ and $Y$ axis, even with the velocity variation of the AR.Drone. Finally, the estimated $Z$ position varies between -1 and 0.5 meters. The change in $Z$ at $t=213$ is caused by the UAV's forward pitch, causing it to move. When the AR.Drone decelerates, we obtain noise in sensor and odometry measurements, so we observe more variability between $t=270$ and $t=295$. The overall performance of joint localization is clearly better than the other methods. 


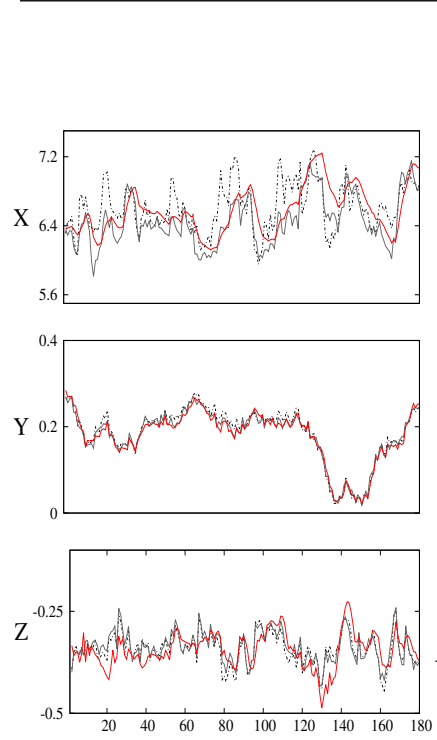

(a)
Localization with no filtering Localization with sensor correction Joint localization
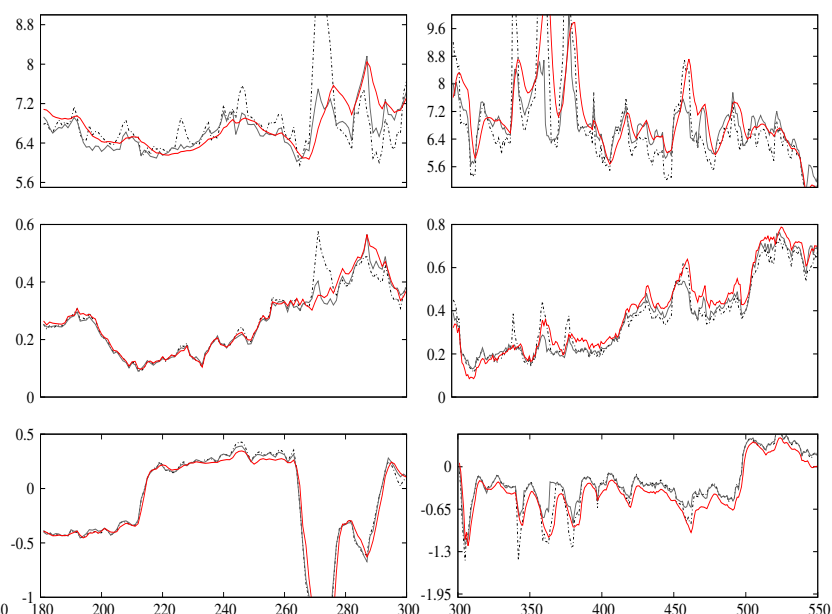

(b)

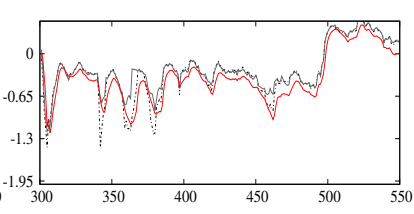

(c)

Fig. 9 Experiment III: outdoor qualitative evaluation. Stability and smoothness of the target's position $(X, Y, Z)$ in the robot coordinate frame where $X$ is forward, $Y$ is left and $Z$ is upward. Each sub figure shows noise on y-axis ranging in meters, while the time $t$ on $x$-axis varies between time 1 and 550, distributed over all columns. (a). Localization when target is stationary and UAV is simply hovering. The peaks at $t=130$ in $Y$ and $Z$ graphs are generated when UAV moves to adjust its position relative to the target. (b). When target is moving and UAV is accelerating at $t=210$ and decelerating at $t=260$ to catch up. (c). When the AR.Drone visual sensor noise increased because of lighting and more clutter background. Higher noise in $X$ shows higher variance due to $2 \mathrm{D}$ bounding box error.

Now we turn to Fig. 9 (c). We observe increased no of spikes in the trajectories caused by error in the $2 \mathrm{D}$ bounding box estimates. This noise results in unstable predictions and more challenging to the proposed joint localization method. The $X$ estimated position of the target varies between 5.4 and 10 meters, the estimated $Y$ position varies between 0 and 0.8 meters and the estimated $Z$ position varies between -1.5 and 0.6 meters. The proposed method performs better than the other two method in terms of stability and smoothness.

\subsection{Experiment IV: Visual tracking}

We tested the visual tracker in both indoor and outdoor environments. In Fig. 10, the first column show outdoor results, captured by AR.Drone. Second column show results of an online pursuit video sequence by Klein et al. [21], and the last two columns show indoor results captured by AR.Drone in a large open room and corridor.

Based on the first frame of each video (R1 in Fig. 10), we initialized CAMSHIFT tracking by manually providing a bounding box for the human target in the scene. We then ran the proposed tracking, suspension, and redetection method to the end of each video. 


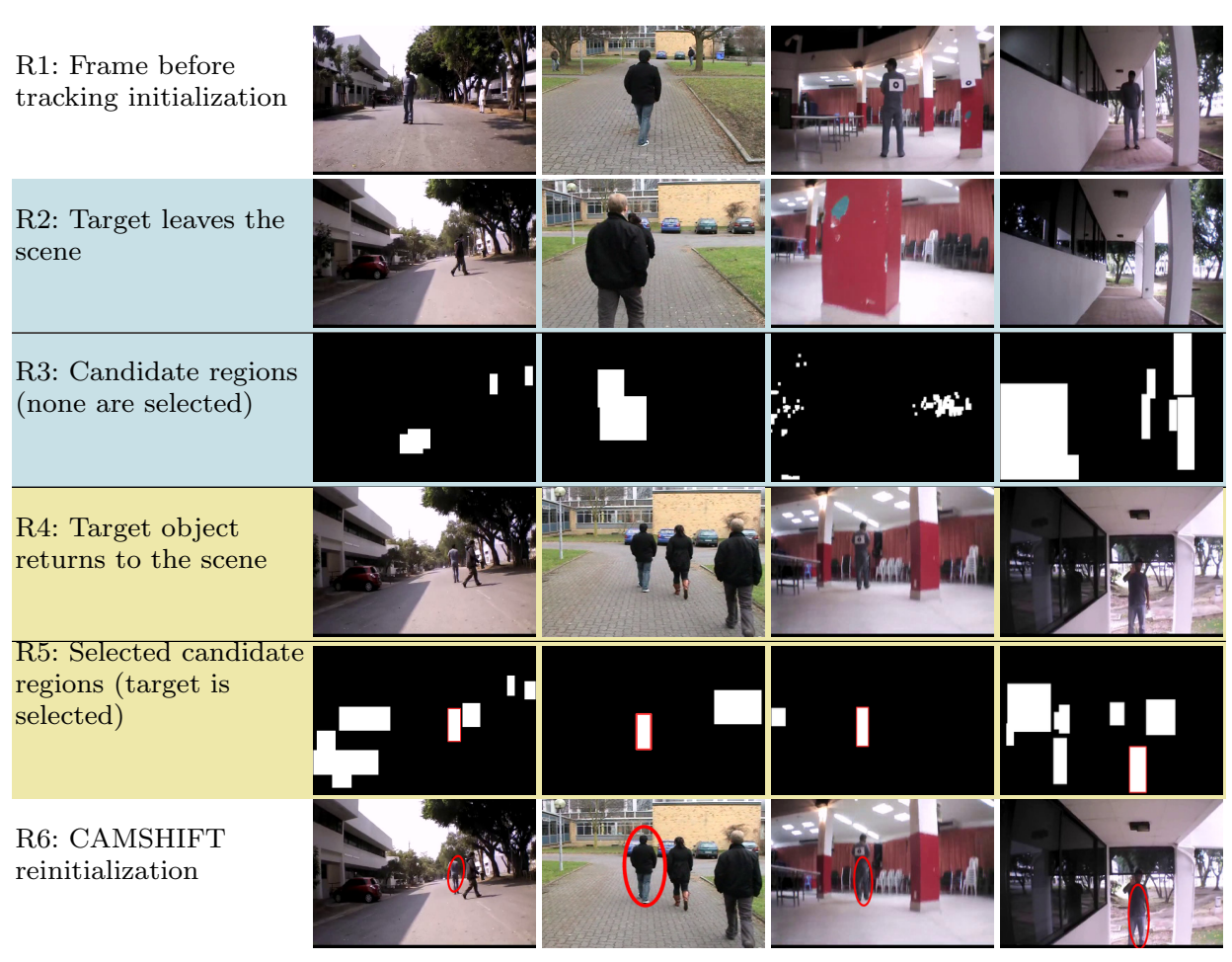

Fig. 10 Experiment IV: The proposed method was tested in different outdoor environments with different background and target objects. Each column shows images from a different video. Rows show results of each step of processing. Blue colored rows show processing when the target is not in the scene. Yellow colored rows show the same processing steps when the target returns to the scene.

During tracking, we incrementally updated the mean $\mu_{t}$ and standard deviation $\sigma_{t}$ of the distance $d_{t}$ between the appearance model $H^{m}$ and the tracked target's color histogram $H_{t}^{r}$. In almost all cases, when the target left the scene, the distance $d_{t}$ exceeded the adaptive threshold, except for a few cases in which the redetection algorithm found a sufficiently similar object in the background.

Rows R2 and R3 in Fig. 10 show example images acquired when the target was absent from the camera field of view. At this point in each video, CAMSHIFT tracking is suspended and the redetection algorithm is running, correctly reporting the absence of the target from the field of view.

Rows R4 and R5 in Fig. 10 show example images acquired after the target has returned to the field of view. The proposed method eventually successfully identifies the candidate region among the possible candidates. In the figure, the selected region is surrounded by a red rectangle.

In each case, CAMSHIFT is correctly reinitialized, as shown in row R6 of Fig. 10 .

Over the four videos, the target was successfully tracked in $95.6 \%$ of the frames in which the target was in the scene, with false positives only $4.4 \%$ of the frames in which the target was not in the scene. The accuracy results on a per video basis are summarized in Table 1. 
Table 1 Experiment IV accuracy measurement: For each video, we report the no of frames the target is visible and occluded or outside FOV, the existence of multiple objects in the image scene, the percentage of frames containing the target in which target was correctly tracked, and the percentage of frames not containing the target in which the target was falsely detected. Incorrect suspension can be calculated by $100 \%$ - true.

\begin{tabular}{c|r|r|c|c|c}
\multirow{2}{*}{ Video } & \multicolumn{2}{|c|}{ Frames w. target } & Multiple & \multicolumn{2}{|c}{ Detection ratio } \\
& present & absent & objects & true & false \\
\hline a & 389 & 50 & Yes & $96.8 \%$ & $2.0 \%$ \\
b & 997 & 20 & Yes & $98.9 \%$ & $0.0 \%$ \\
c & 541 & 150 & No & $88.8 \%$ & $5.3 \%$ \\
d & 426 & 174 & No & $97.3 \%$ & $3.4 \%$
\end{tabular}

We tested the runtime performance of the system on two different hardware configurations, a 2.26 GHz Intel Core i3 laptop running 32-bit Ubuntu Linux 11.10 and a 1.6 GHz Intel Atom N280 single core netbook running 32-bit Lubuntu 11.10. The results are summarized in Table 2. Both algorithms run at high frame rates, with the worst case of just over $10 \mathrm{fps}$ for redetection on the Atom processor. The method is clearly feasible for onboard execution by a mobile robot with modest computational resources.

Table 2 Experiment IV: Runtime performance of visual tracking and redetection algorithms on two different processors.

\begin{tabular}{c|c|c|c}
\multicolumn{2}{c|}{ Redetection Phase } & \multicolumn{2}{c}{ Tracking Phase } \\
Core i3 & Atom N280 & Core i3 & Atom N280 \\
\hline $41.455 \mathrm{~ms}$ & $91.232 \mathrm{~ms}$ & $16.340 \mathrm{~ms}$ & $49.234 \mathrm{~ms}$
\end{tabular}

\section{Conclusion}

In this paper, we propose a joint localization method that fuses target dynamics and UAV kinematics into one state space model, and we demonstrate how to integrate sensor measurements from a moving monocular camera into the resulting filter. We show that joint localization produces more stable, smooth, and noiseresistant trajectories than those produced by standard filters. The joint localization filter performs well even when there are sudden changes in the sensor measurement indicating an erroneous detection or a rapid change of the target object's position.

We have also proposed a computationally efficient and accurate visual tracker that can deal with situations where the object disappears from the field of view. The target's 3D position is inferred by the tracker from the bounding box of its projection into the $2 \mathrm{D}$ image. The result is an estimated trajectory sufficiently smooth to use in a pursuit robot. In a series of experiments, we show that the proposed filtering technique outperforms traditional filtering methods.

In future work, we plan to further investigate adaptive and discriminative modeling of the target and the background, to address situations in which the target's appearance varies as it moves through areas with different lighting conditions and backgrounds. 


\section{Acknowledgment}

AB was supported by graduate fellowships from the University of Balochistan, Quetta, the Higher Education Commission of Pakistan, and the Asian Institute of Technology, Thailand. TK was supported by EU-ICT project 600623 'STRANDS' and CZ-MSMT project LH11053. We thank Niraj Shakya for help with experimental data collection.

\section{References}

1. Allen, J.G., Xu, R.Y.D., Jin, J.S.: Object Tracking using CamShift Algorithm and Multiple Quantized Feature Spaces. In: Pan-Sydney Area Workshop on Visual Information Processing, vol. 36, pp. 3-7 (2004)

2. Barrientos, A., Colorado, J., del Cerro, J., Martinez, A., Rossi, C., Sanz, D., Valente, J.: Aerial remote sensing in agriculture: A practical approach to area coverage and path planning for fleets of mini aerial robots. Journal of Field Robotics 28(5), 667-689 (2011)

3. Basit, A., Dailey, M.N., Lakanacharoen, P., Moonrinta, J.: Fast target redetection for CAMSHIFT using back-projection and histogram matching. In: International Conference on Computer Vision Theory and Applications (VISAPP) (2014)

4. Basit, A., Dailey, M.N., Laksanacharoen, P.: Model driven state estimation for target pursuit. In: IEEE International Conference on Control, Automation, Robotics \& Vision, pp. 1077-1082 (2012)

5. Bi, Y., Duan, H.: Implementation of autonomous visual tracking and landing for a low-cost quadrotor. Optik - International Journal for Light and Electron Optics 124(18), 3296 3300 (2013)

6. Bradski, G.: Real Time Face and Object Tracking as a Component of a Perceptual User Interface. In: IEEE Workshop on Applications of Computer Vision, 1998., pp. 214-219 (Oct)

7. Bristeau, P.J., Callou, F., Vissière, D., Petit, N., et al.: The Navigation and Control technology inside the AR.Drone micro UAV. In: 18th IFAC World Congress, pp. 1477-1484 (2011)

8. Chen, H., Houkes, Z.: Model-based recognition and classification for surface texture of vegetation from an aerial sequence of images. In: Proceedings of SPIE, vol. 3222, pp. 236-245 (1997)

9. Comaniciu, D., Ramesh, V., Meer, P.: Real-Time Tracking of Non-Rigid Objects using Mean Shift. In: IEEE Conference on Computer Vision and Pattern Recognition, vol. 2, pp. $142-149(2000)$

10. Comaniciu, D., Ramesh, V., Meer, P.: Kernel-based object tracking. IEEE Transactions on Pattern Analysis and Machine Intelligence 25(5), 564-577 (2003)

11. Davison, A.J., Reid, I.D., Molton, N.D., Stasse, O.: MonoSLAM: Real-time single camera SLAM. IEEE Transactions on Pattern Analysis and Machine Intelligence 29(6), 1052-1067 (2007)

12. Denman, S., Chandran, V., Sridharan, S.: An adaptive optical flow technique for person tracking systems. Pattern Recognition Letters 28(10), 1232-1239 (2007)

13. Funk, N.: A study of the Kalman filter applied to visual tracking. Tech. Rep. CMPUT, University of Alberta (2003)

14. Gupte, S., Mohandas, P., Conrad, J.: A survey of quadrotor unmanned aerial vehicles. In: IEEE Southeastcon 2012, pp. 1-6 (2012)

15. Gktoan, A., Sukkarieh, S., Bryson, M., Randle, J., Lupton, T., Hung, C.: A rotary-wing unmanned air vehicle for aquatic weed surveillance and management. Journal of Intelligent and Robotic Systems 57(1-4), 467-484 (2010)

16. Herwitz, S., Johnson, L., Dunagan, S., Higgins, R., Sullivan, D., Zheng, J., Lobitz, B., Leung, J., Gallmeyer, B., Aoyagi, M., et al.: Imaging from an unmanned aerial vehicle: Agricultural surveillance and decision support. Computers and Electronics in Agriculture 44(1), 49-61 (2004)

17. Higuchi, K., Shimada, T., Rekimoto, J.: Flying sports assistant: External visual imagery representation for sports training. In: 2nd Augmented Human International Conference (AH), 2011, pp. 7:1-7:4 (2011) 
18. Jiménez-Berni, J.A., Zarco-Tejada, P.J., Suarez, L., Fereres, E.: Thermal and narrowband multispectral remote sensing for vegetation monitoring from an unmanned aerial vehicle. IEEE Transactions on Geoscience and Remote Sensing 47(3), 722-738 (2009)

19. Karavasilis, V., Nikou, C., Likas, A.: Visual tracking by adaptive Kalman filtering and mean shift. In: Artificial Intelligence: Theories, Models and Applications, pp. 153-162. Springer (2010)

20. Kim, J., Shim, D.: A vision-based target tracking control system of a quadrotor by using a tablet computer. In: 2013 International Conference on Unmanned Aircraft Systems (ICUAS), pp. 1165-1172 (2013)

21. Klein, D.A., Schulz, D., Frintrop, S., Cremers, A.B.: Adaptive Real-Time Video-Tracking for Arbitrary Objects. In: IEEE Int. Conf. on Intelligent Robots and Systems (IROS), pp. $772-777(2010)$

22. Krajník, T., Nitsche, M., et al.: External Localization System for Mobile Robotics. In: International Conference on Advanced Robotics. IEEE, Montevideo (2013)

23. Lan, Y., Thomson, S.J., Huang, Y., Hoffmann, W.C., Zhang, H.: Review: Current status and future directions of precision aerial application for site-specific crop management in the USA. Computer and Electronics in Agriculture 74(1), 34-38 (2010)

24. Lou, J., Yang, H., Hu, W.M., Tan, T.: Visual vehicle tracking using an improved EKF. In: Asian Conference of Computer Vision (ACCV), pp. 296-301 (2002)

25. Murphy, D.W., Cycon, J.: Applications for mini VTOL UAV for law enforcement. In: Enabling Technologies for Law Enforcement and Security, pp. 35-43. International Society for Optics and Photonics (1999)

26. Perreault, S., Hebert, P.: Median filtering in constant time. Image Processing, IEEE Transactions on 16(9), 2389-2394 (2007)

27. Porikli, F.: Integral histogram: a fast way to extract histograms in cartesian spaces. In: Computer Vision and Pattern Recognition, 2005. CVPR 2005. IEEE Computer Society Conference on, vol. 1, pp. 829-836 (2005)

28. Pupilli, M., Calway, A.: Real-time camera tracking using a particle filter. In: British Machine Vision Conference (2005)

29. Shimin, F., Qing, G., Sheng, X., Fang, T.: Human tracking based on mean shift and Kalman filter. In: Artificial Intelligence and Computational Intelligence, vol. 3, pp. 518$522(2009)$

30. Sizintsev, M., Derpanis, K., Hogue, A.: Histogram-based search: A comparative study. In: Computer Vision and Pattern Recognition, 2008. CVPR 2008. IEEE Conference on, pp. 1-8 (2008)

31. Ta, D.N., Chen, W.C., Gelfand, N., Pulli, K.: Surftrac: Efficient tracking and continuous object recognition using local feature descriptors. In: IEEE Conference on Computer Vision and Pattern Recognition., pp. 2937-2944 (2009)

32. Welch, G., Bishop, G.: An introduction to the kalman filter. Tech. rep., Chapel Hill, NC, USA (1995)

33. Yokoyama, M., Poggio, T.: A contour-based moving object detection and tracking. In: Visual Surveillance and Performance Evaluation of Tracking and Surveillance., pp. 271$276(2005)$

34. Zhou, H., Yuan, Y., Shi, C.: Object tracking using SIFT features and mean shift. Computer Vision and Image Understanding 113(3), 345-352 (2009) 